\title{
The LHC Machine
}

\section{Lyndon Evans}

CERN

CH-1211 Geneva 23, Switzerland

E-mail: lyn.evans@cern.ch

The 2009 Europhysics Conference on High Energy Physics,

Krakow , Poland

July 16-22, 2009 


\section{Introduction}

The first time that the LHC design was presented at a HEP conference was in Glasgow in 19941). Table 1 shows a short parameter list presented there. It is remarkable how little the LHC design has changed since then. The only parameter in that table that is no longer valid is the dipole field at $7 \mathrm{TeV}$. Optimization of the space in the interconnects has allowed the magnets to be made slightly longer and thereby the field to be reduced from 8.65T to 8.33T. The other change has been that CMS was moved from Point 6, where it would have required the premature closure of the LEP detector OPAL to Point 5.

Table 1 - LHC main parameters.

\begin{tabular}{|lr|l|}
\hline Energy & $(\mathrm{TeV})$ & 7.0 \\
\hline Dipole field & $(\mathrm{T})$ & 8.65 \\
\hline Luminosity & $\left(\mathrm{cm}^{-2} \mathrm{~s}^{-1}\right)$ & $10^{34}$ \\
\hline Beam-beam parameter & & 0.0032 \\
\hline Injection energy & $(\mathrm{GeV})$ & 450 \\
\hline Circulating current/beam & $(\mathrm{A})$ & 0.53 \\
\hline Bunch spacing & $(\mathrm{ns})$ & 25 \\
\hline Particles per bunch & & $1 \times 10^{11}$ \\
\hline Stored beam energy & $(\mathrm{MJ})$ & 332 \\
\hline Normalized transverse emittance & $(\mu \mathrm{m})$ & 3.75 \\
\hline R.m.s. bunch length & $(\mathrm{m})$ & 0.075 \\
\hline Beta values at l.P. & $(\mathrm{m})$ & 0.5 \\
\hline Crossing angle & $(\mu \mathrm{rad})$ & 200 \\
\hline Beam lifetime & $(\mathrm{h})$ & 22 \\
\hline Luminosity lifetime & $(\mathrm{h})$ & 10 \\
\hline Energy loss per turn & $(\mathrm{keV})$ & 6.9 \\
\hline Critical photon energy & $(\mathrm{eV})$ & 45.6 \\
\hline Total radiated power per beam & $(\mathrm{kW})$ & 3.7 \\
\hline
\end{tabular}

\section{Machine cooldown}

2008 was a very eventful year for the LHC. During the first half of the year, the whole machine was cooled down (Figure 1). From room temperature to $80 \mathrm{~K}$ the helium circulating in the magnets is cooled down by vapourising liquid nitrogen in a heat exchanger. In total, 1200 tons of LN2 is needed for a single sector, the whole process taking about 10 days with 60 trucks, each containing 20 tons of LN2 arriving every 4 hours. Between $80 \mathrm{~K}$ and $4.5 \mathrm{~K}$, the helium refrigerators are used. Finally the cold compressors producing helium at $15 \mathrm{mbar}$ pressure are switched on to reduce the temperature to the operating value of $1.9 \mathrm{~K}$. 


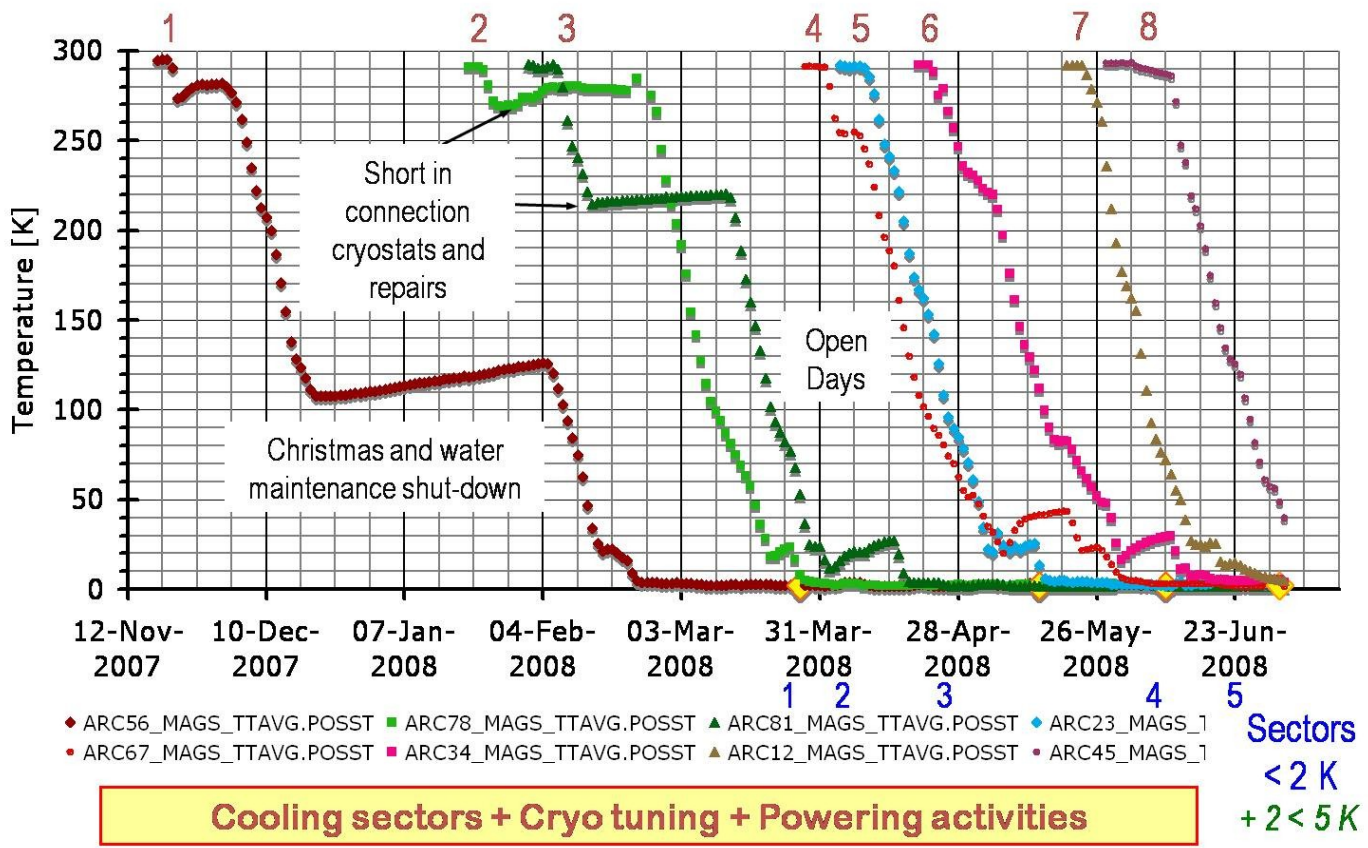

Figure 1 - Cooldown of LHC sectors.

\section{First commissioning}

By the $10^{\text {th }}$ September 2008, seven of the eight sectors had been successfully commissioned to $5.5 \mathrm{TeV}$ in preparation for a run at $5 \mathrm{TeV}$. Due to lack of time, the eighth sector had only been taken to $4 \mathrm{TeV}$. Beam commissioning started by threading beam 2 (Figure 2) around the ring, stopping it at each long straight section sequentially in order to correct the trajectory. In less than an hour the beam had completed a full turn, witnessed by a second spot on a fluorescent screen intercepting both injected and circulating beams (Figure 3).

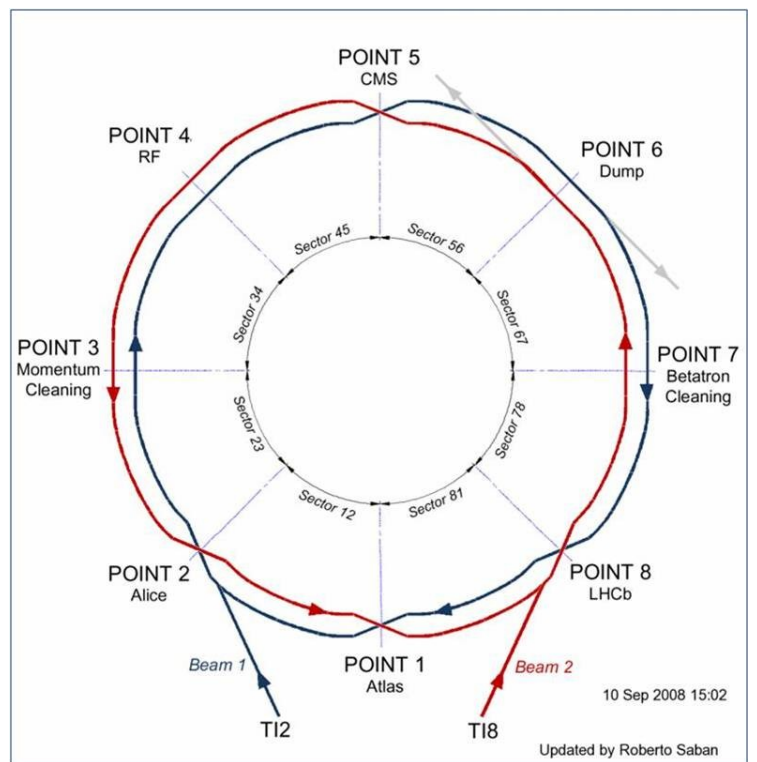


Figure 2 - LHC beam layout.

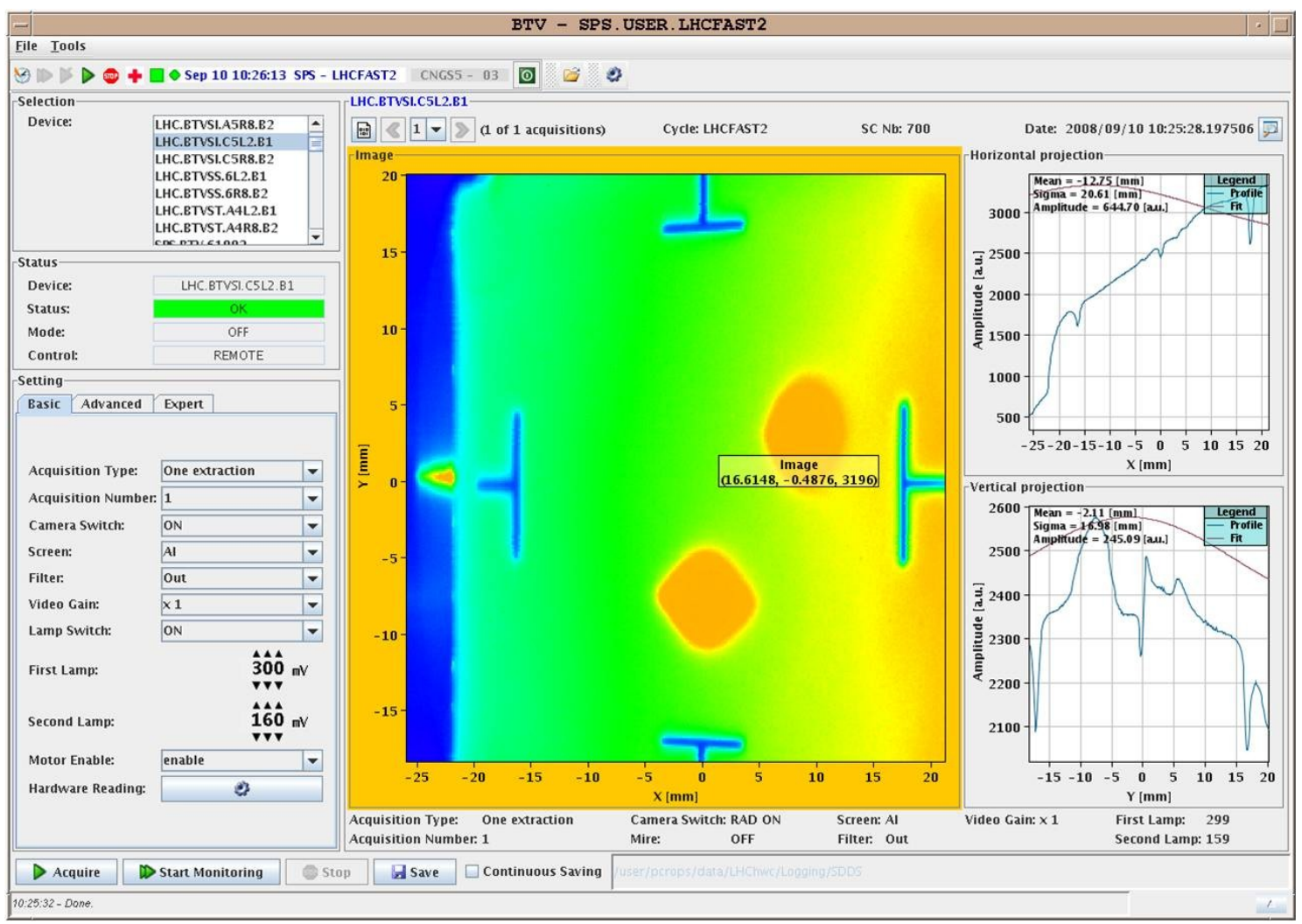

Figure 3 - Beam on turns 1 and 2.

Very quickly, a beam circulating for a few hundred turns could be established (Figure 4). The decay in intensity is due to the debunching of the beam around the ring since the Radiofrequency system was not yet switched on. Figures 5 to 8 show the RF capture process. Each horizontal line on the mountain range display records the bunch intensity every 10 turns. Without the RF the beam debunches as it should in about 250 turns, or $25 \mathrm{msec}$. In the next figure, the first attempt was made to capture the beam, but as can be seen, the injection phase was completely wrong. Adjusting the phase allowed a partial capture, but at a slightly wrong frequency. Finally, adjusting the frequency resulted in a perfect capture. 


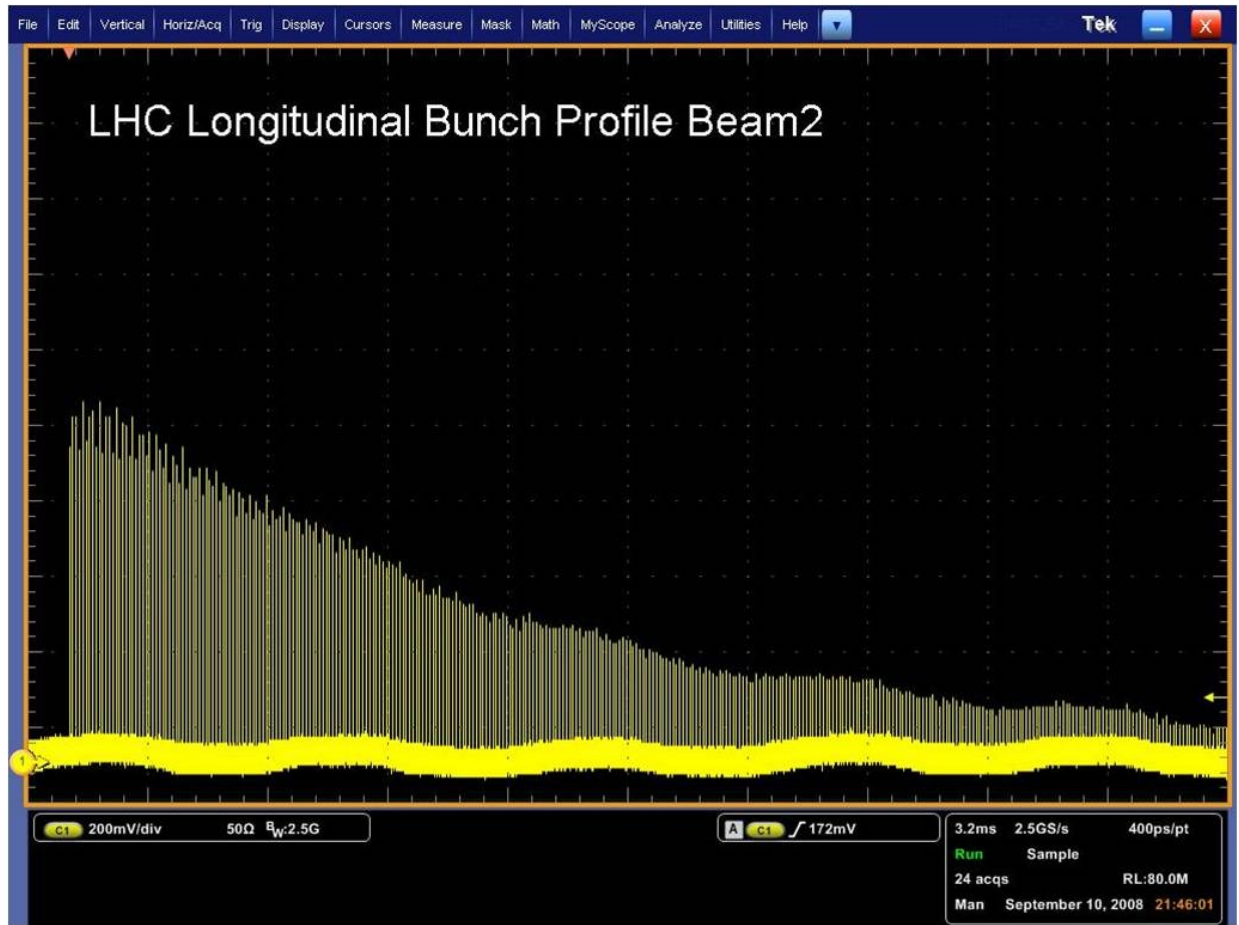

Figure 4 - Few 100 turns.

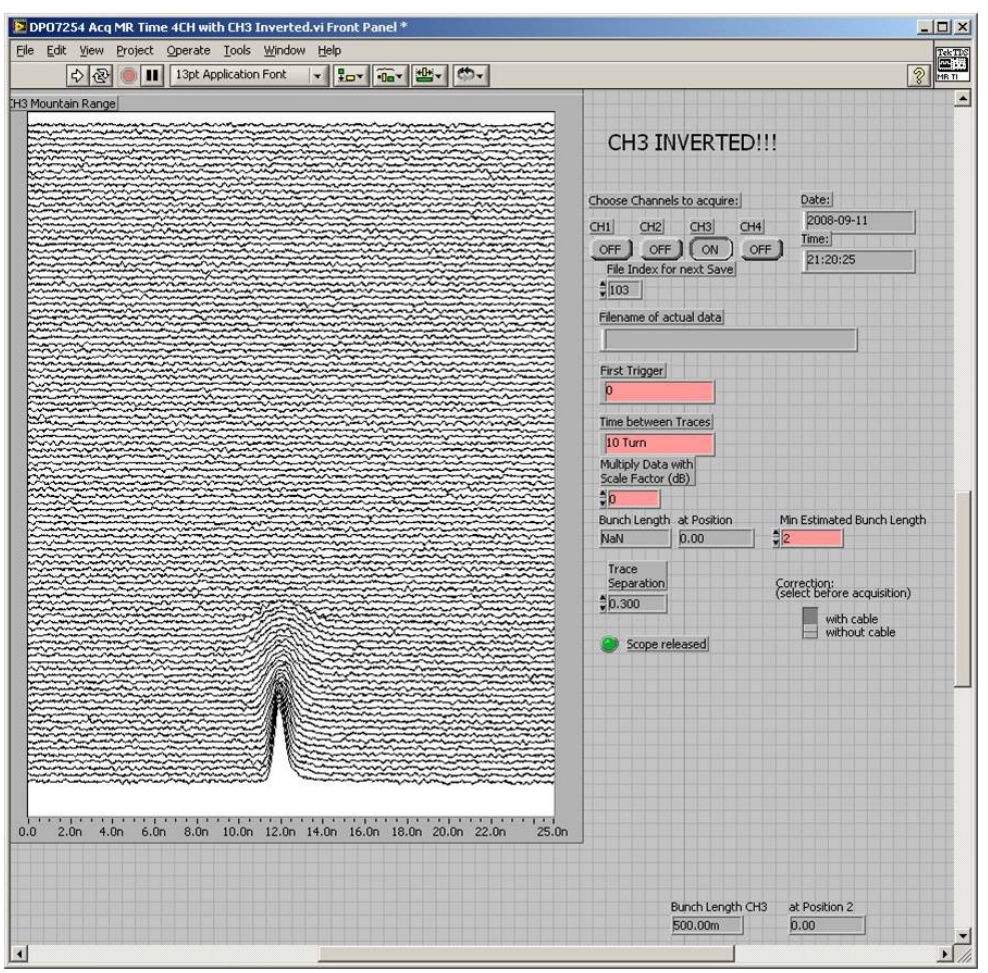

Figure 5 - No RF, debunching in $\sim 25 * 10$ turns, i.e. roughly $25 \mathrm{~ms}$. 


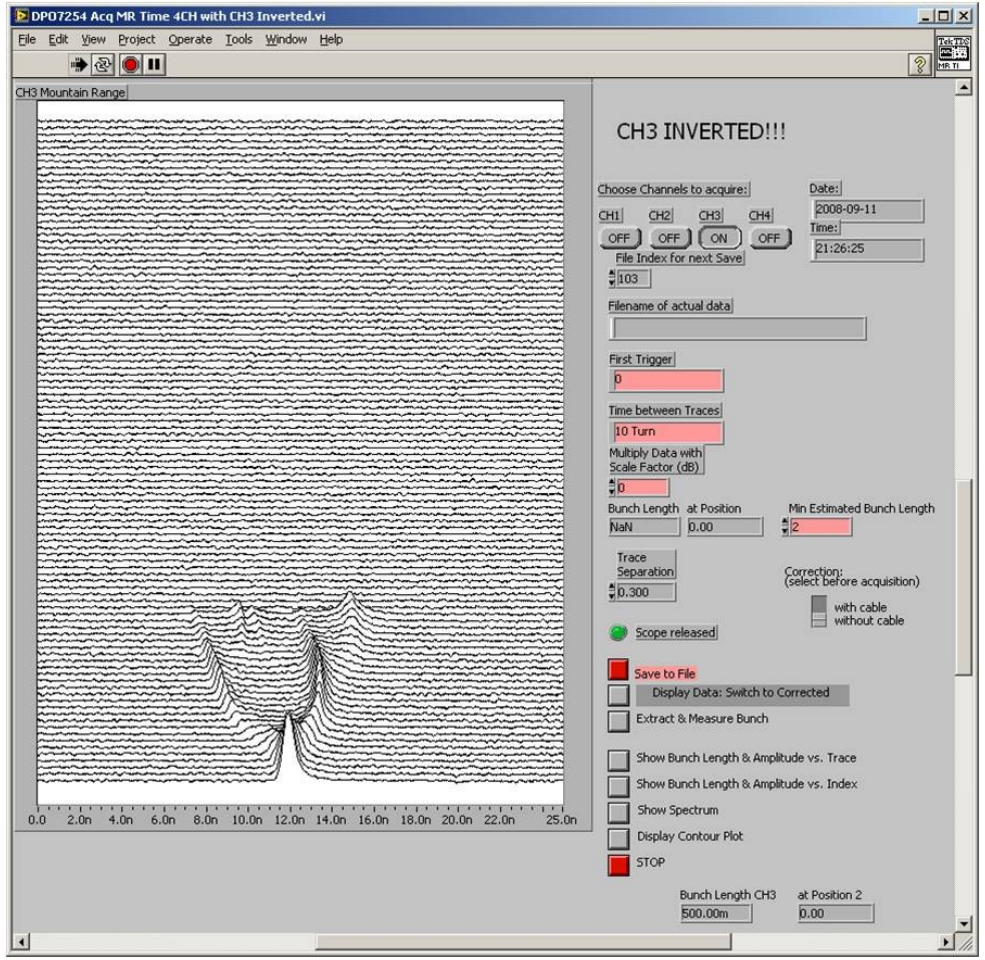

Figure 6 - First attempt at capture, at exactly the wrong injection phase.

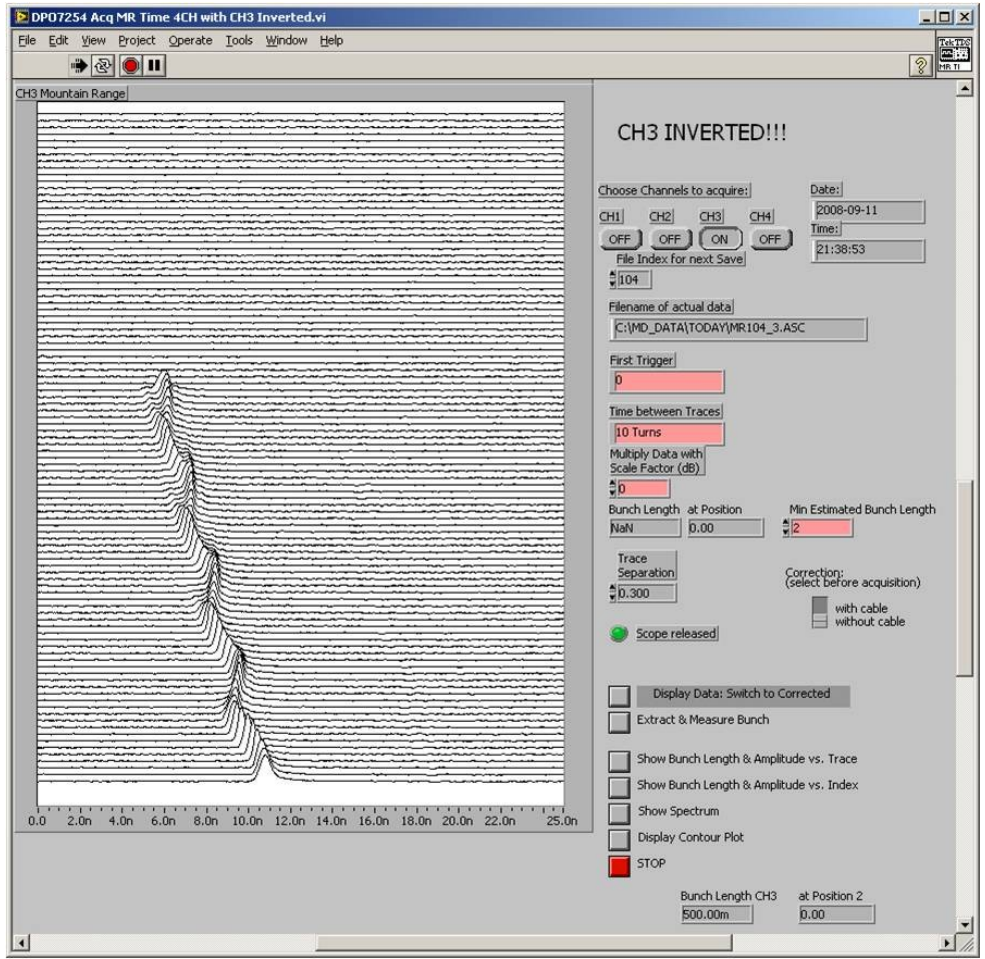

Figure 7 - Capture with corrected injection phasing. 


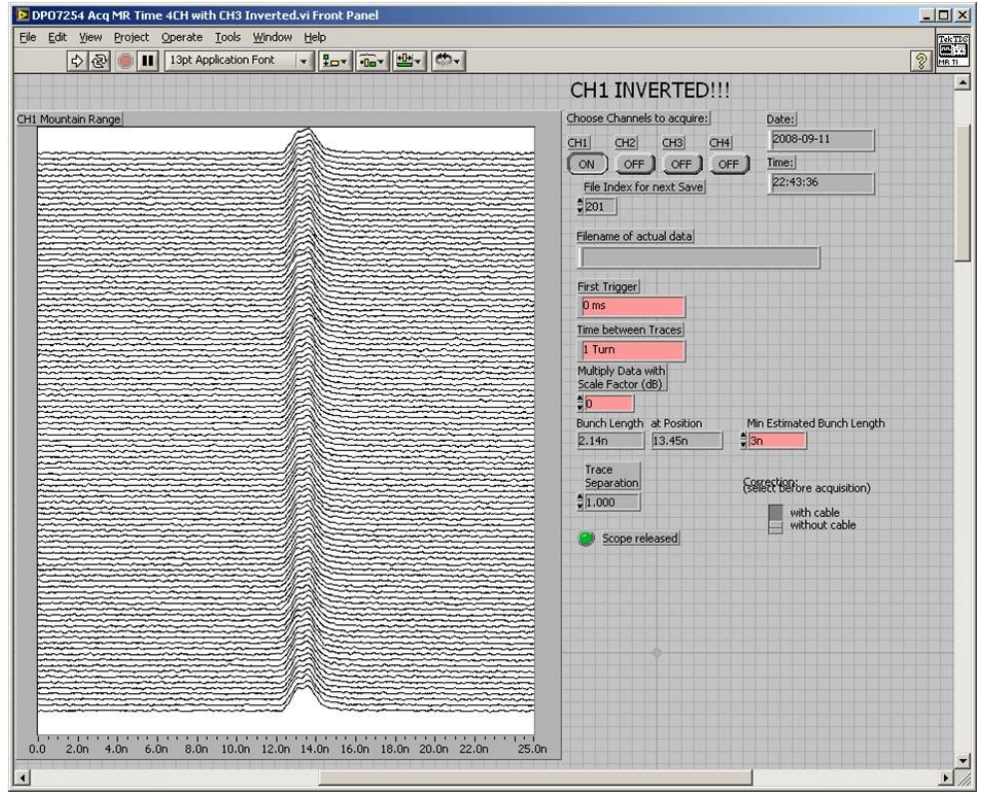

Figure 8 - Capture with optimum injection phasing, correct reference.

The closed orbit could then be corrected. Figure 9 shows the first orbit correction where remarkably at this early stage, the rms orbit is less than $2 \mathrm{~mm}$. It can be seen that in the horizontal plane the mean orbit is displaced radially by about a millimeter, indicative of an energy mismatch of about 0.9 permil.

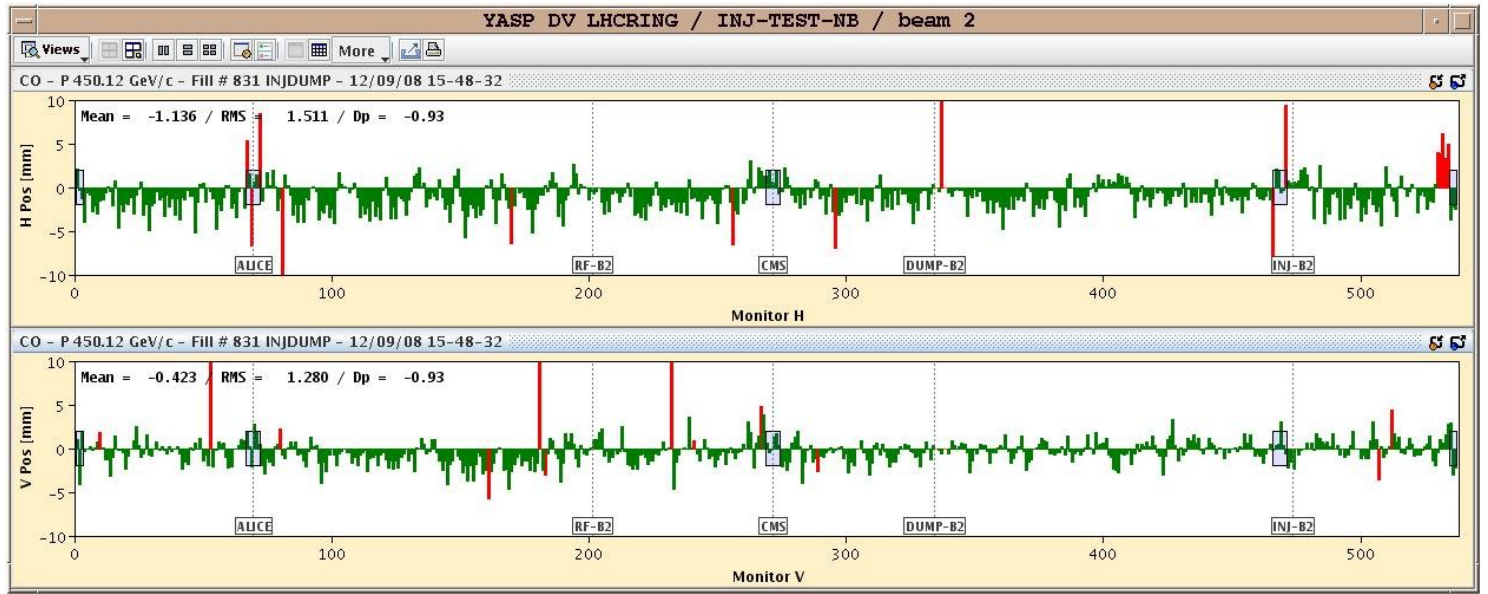

Figure $9-$ Corrected closed orbit on B2. Energy offset of $\sim-0.9$ permil due to the capture frequency.

\section{The accident}

Commissioning proceeded rapidly with circulating beam in the other ring until, on $18^{\text {th }}$ September a transformer failed at Point 8 , taking down the cryogenics in that sector. Since it was impossible to circulate beam, attention turned to bringing the last remaining sector up to 
to. $5 \mathrm{TeV}$ like the others. On $19^{\text {th }}$ September, the last remaining circuit was being ramped to full field when, at 5.2 TeV a catastrophic rupture of a busbar occurred causing extensive damage in Sector 34. These busbars are connected by induction brazing with three layers of Tin/Silver solder in a copper box. Initially it was foreseen to clamp these busbars mechanically, but this was discarded on the recommendation of an external review committee on the grounds that it would increase the hydraulic impedance in the interconnect region. A fact finding commission was established where it was concluded that the most probable cause of the accident was too high a resistivity in a superconducting joint due to the omission of the solder.

\section{Diagnostics}

An urgent priority after the accident was to sift through most mortem data to see if any precursors of the accident could be detected, in particular any anomalous temperature increase in the affected area.

Detecting temperature rise in the superfluid helium is made difficult by two factors. The first is the enormous thermal conductivity of superfluid helium (Figure 10). This provides good cooling of joints initially, but the thermal conductivity is a function of flux density (Figure 11), so as the heating increases, the cooling capacity quickly collapses, especially in the region of the splices with high hydraulic impedance.

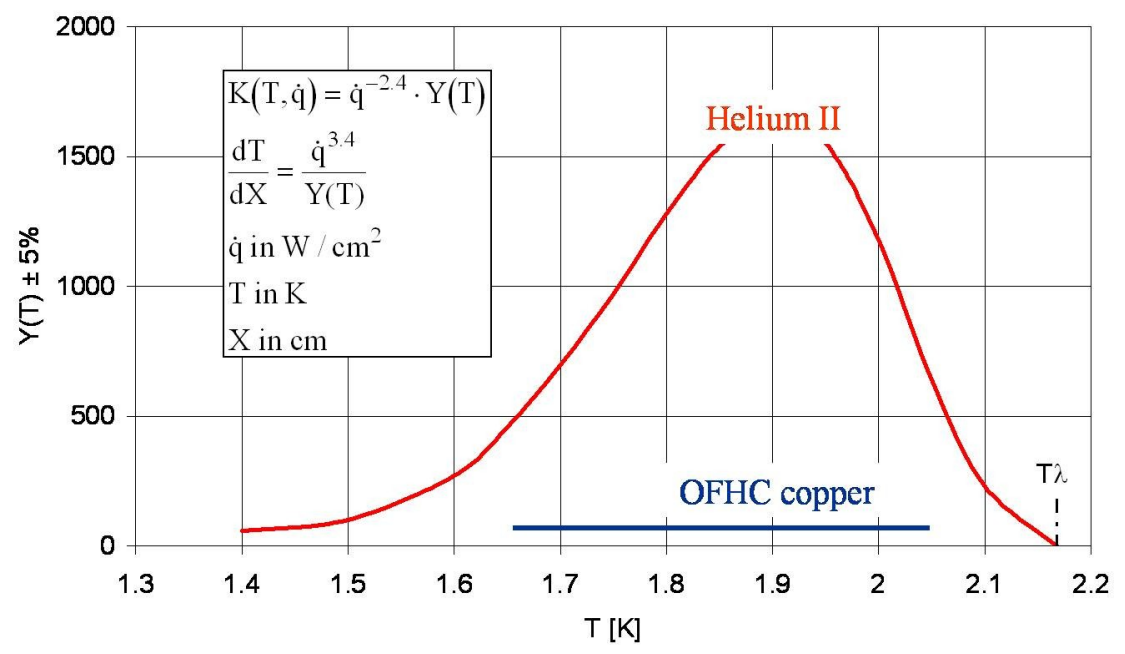

Figure 10 - Equivalent thermal conductivity of He II. 


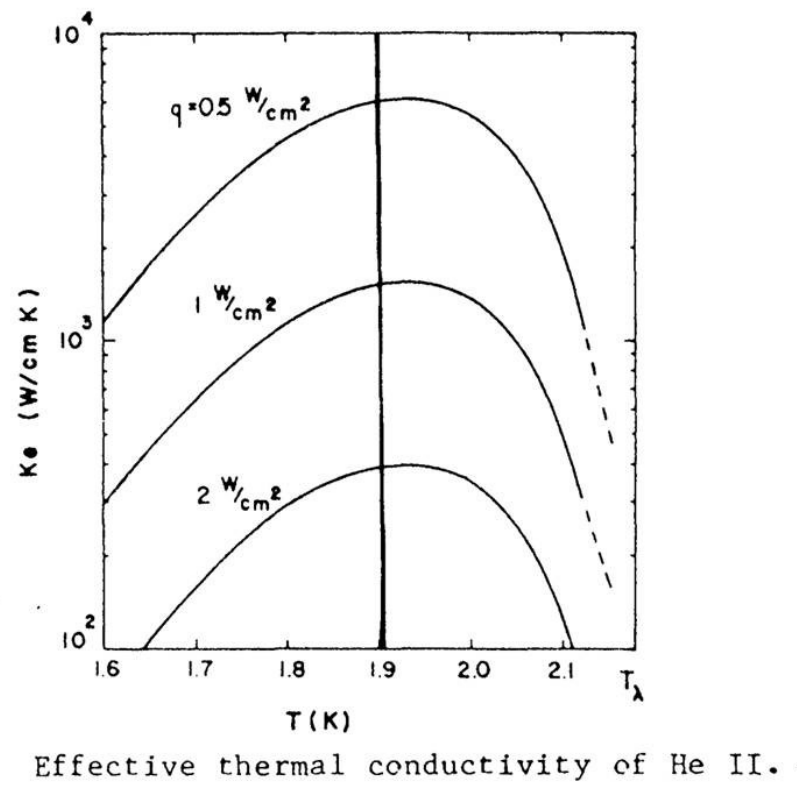

Figure 11

The other reason why it was impossible to observe a temperature rise was the configuration of the superfluid cooling circuits themselves. Figure 12 shows one cryogenic cell containing two $106 \mathrm{~m}$ long periods of the machine. The primary superfluid flows through bayonet heat exchangers, the flow rate being controlled through Joule-Thomson valves (CV910 in the diagram). These valves are in a servo loop which keeps the temperature constant.

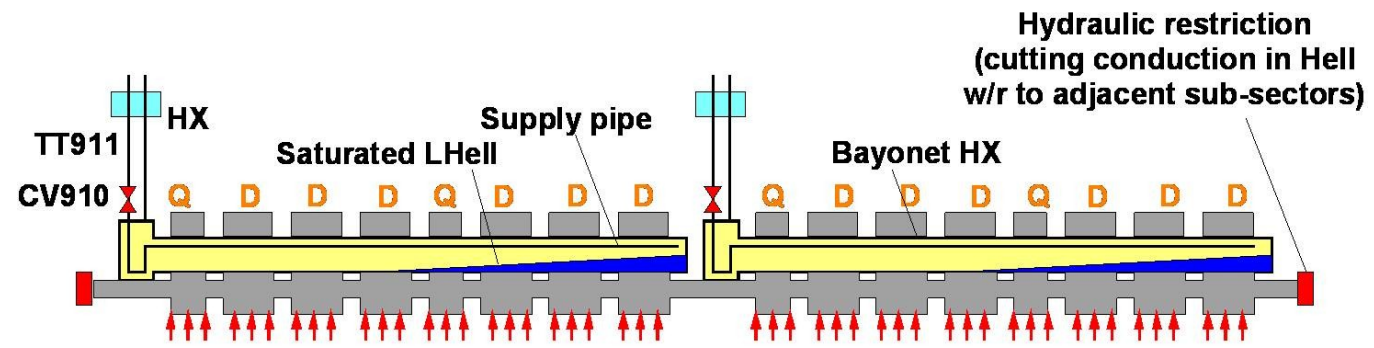

\section{Principle: \\ $\rightarrow$ Blocking of the JT valve (CV910) at a value to extract the static heat inleaks before the powering $\rightarrow$ Then, the temperature drift is mainly due to electrical resistive heating dissipated during the powering}

Figure 12 - Sub-sector magnet cooling scheme.

It was obviously very important to find a way to be sure that there are no more such bad joints in the machine. Two methods were developed. The first of these relied on calorimetry. 
With the servo loops open, the valves could be adjusted to just balance the static heat inleak. Under these conditions it was shown that a calibrated heat inleak of $10 \mathrm{~W}$ through a resistor could be detected and by measuring the rate of temperature rise (Figure 13), the original $10 \mathrm{~W}$ could be reconstituted purely calorimetrically. Note the temperature axis with $5 \mathrm{mK}$ ticks! Once this calibration was made, a sector was powered to $5 \mathrm{kA}$ with the J-T valves in open loop. The normal signal to be expected during a current cycle is a slight heating during ramp and deramp due to eddy currents and slow cooling on flat top. Figure 14 shows a cell in which this was not the case. The slow monatonic heating on flat top was consistent with a 100 nano-Ohm resistance somewhere in the cell.

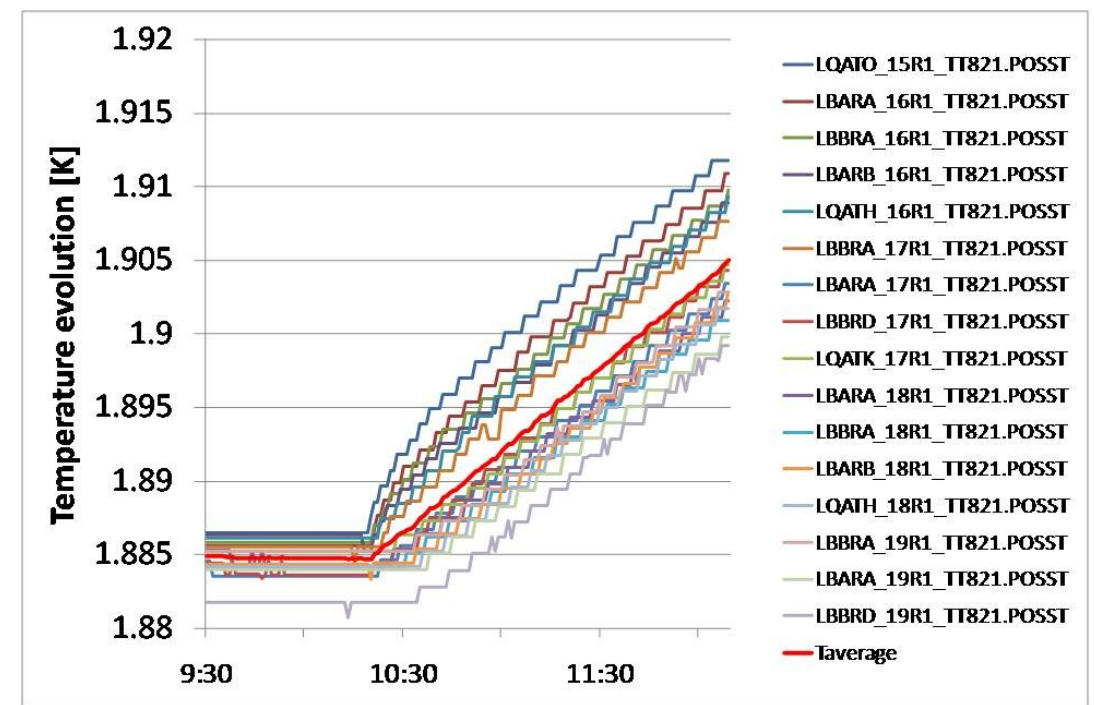

Figure 13 - Experimental validation: temperature evolution.

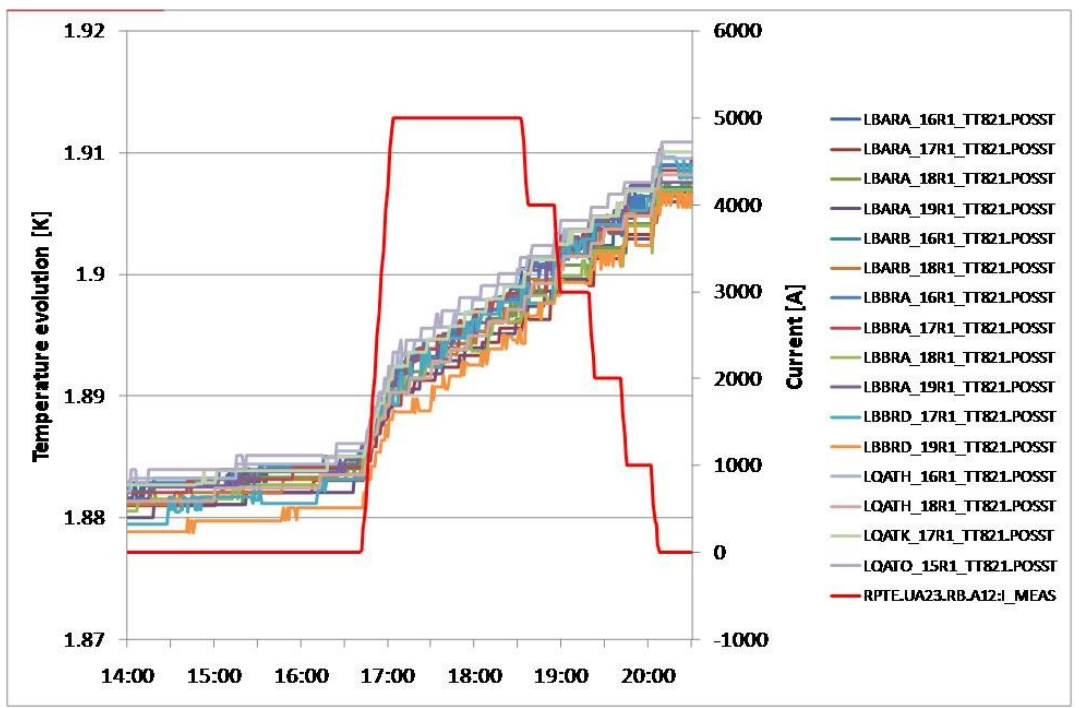

Figure 14 - Powering example: 15R1 powering @ 5000A. 
Every magnet is equipped with a "post mortem" card containing an ADC and a buffer memory in order to measure voltages, usually on a trigger due to a quench. It was realized that these cards could also be used to improve the signal-to-noise ratio in measuring voltages in DC conditions by averaging, thereby opening up the possibility of making Ohmic measurements across each splice. Figure 15 shows such a measurements of all the joints in the dipole chains of Sectors 67 and 78 during a stepwise current ramp to $5 \mathrm{kA}$. In Sector 67, there is one anomaly visible with a resistance of 47 nano-Ohms. It was possible to locate exactly which splice was responsible. Both the 100 nano-Ohm splice previously mentioned and the 47 nano-Ohm splice were inside magnets which had already been tested to full current. They have both been removed and the bad splices confirmed. No other such splices have been detected anywhere else in the machine.

Results from provoked massive Post-Mortem of all dipoles in sectors $67 \& 78$

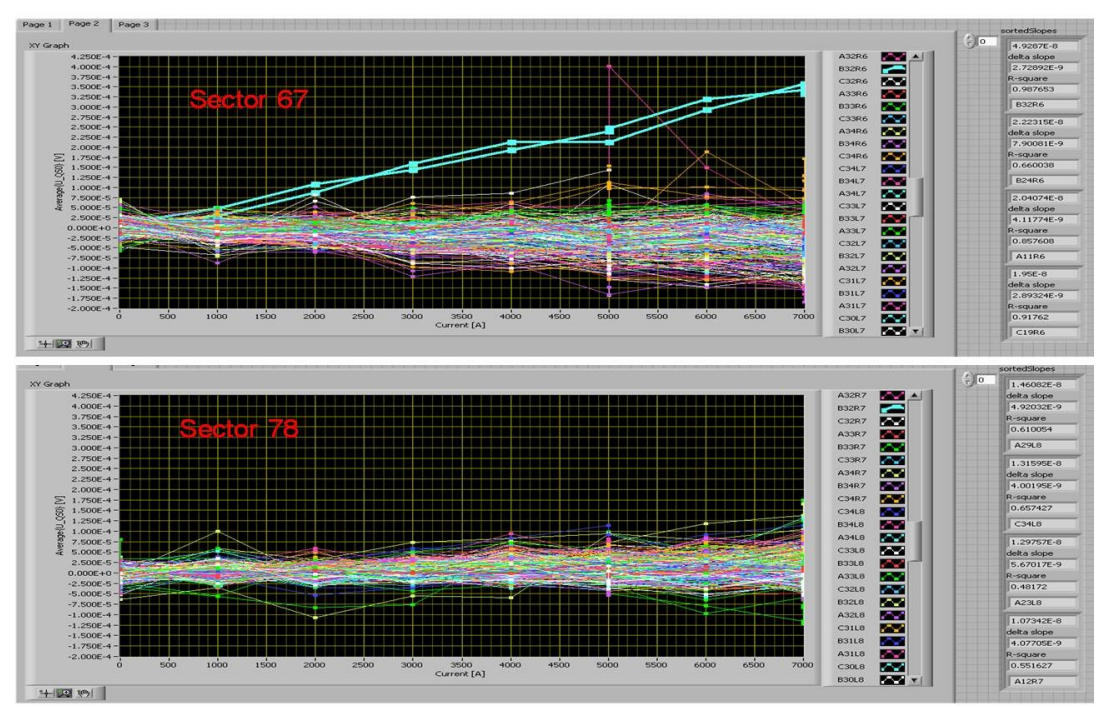

Figure 15 - Snapshots in S67 and S78 on all 154 dipoles - B32.R6 with a high (47 n $\Omega)$ joint resistance between the poles of one aperture.

\section{Conclusions}

Initial commissioning of the LHC went extremely smoothly. Circulating and captured beam were achieved in record time. The 2-in-1 structure of the magnets works exactly as predicted. The machine optics already looks extremely good with the closed orbit corrected to less than $2 \mathrm{~mm}$ rms.

The unfortunate splice incident has created a lot of damage which has to be repaired. Two powerful diagnostic tools have been developed to detect bad splices and to allow a permanent monitoring during operation. The repair is estimated to take about 9 months. The LHC should be back in operation in Autumn 2009. 